\title{
Noise Model Analysis on Image Sensor Arrays
}

\author{
Gwanggil Jeon \\ Department of Embedded Systems Engineering, Incheon National University \\ 119 Academy-ro, Yeonsu-gu, Incheon 406-772, Korea \\ gjeon@incheon.ac.krsss
}

\begin{abstract}
This paper presents a new noise model and its application to Bayer color filter array (CFA). We investigate the effect of various noise models. In addition, we study three possible Bayer patterns. For example, current Bayer CFA utilizes RGGB pattern. However, one may consider other color combinations such as RRGB or RGBB patterns. To prove $R G G B$ pattern's superiority, we investigate each pattern in various noise model and noise level. We also studied the method how to generate noisy image from noisy free image. Traditional noise models do not take into account white balance, gamma and tone correction issues. Simulation results indicate that the new noise model is more reasonable than the conventional models.
\end{abstract}

Keywords: Noise model, color image, color interpolation, color combination

\section{Introduction}

The light was studied by Newton who explained that white light includes every possible spectral colors [1-2]. Therefore, if all colored lights are added, it becomes white color. In contrast, when specific spectral color is excluded from the white light, complementary color is revealed [3-6]. In general, to generate certain color image, it is required to have three colors: red, green, and blue. These three colors are noted as primary colors. There are two color rules to generate colors. One is additive color and the other one is subtractive color. The former one is used for blending lights. In contrast, subtractive color is used by subtracting parts of the spectrum of light. The subtractive color is used for painting, dying, and inks.

For additive color system, the mixing of two or more primary colors in equal ratio will generate new colors [7]. For example, red and green generate yellow, red and blue generate magenta, and green and blue generate cyan [8]. In contrast, blue is generated by mixing magenta and cyan, green is generated by mixing yellow and cyan, and red is generated by mixing yellow and magenta [9-11]. Figure 1(a) shows the consequent color outcome of blending of three primary colors. The color of an object is given by materials. This is ruled by subtractive color, and the remaining light is returned to human eyes (Figure. 1b). Figure 1(c) implies a method to obtain certain color. For example, to obtain yellow, magenta, and cyan, one may take away blue, green, and red from white light.

In this paper, we propose a new noise model and study its effects on Bayer CFA [12]. The remainder of this paper is as follows. Section 2 explains noise model. Section 3 explains possible color combinations for Bayer pattern. Section 4 describes simulation results and discussion. Finally, conclusion remarks are shown in Section 5. 


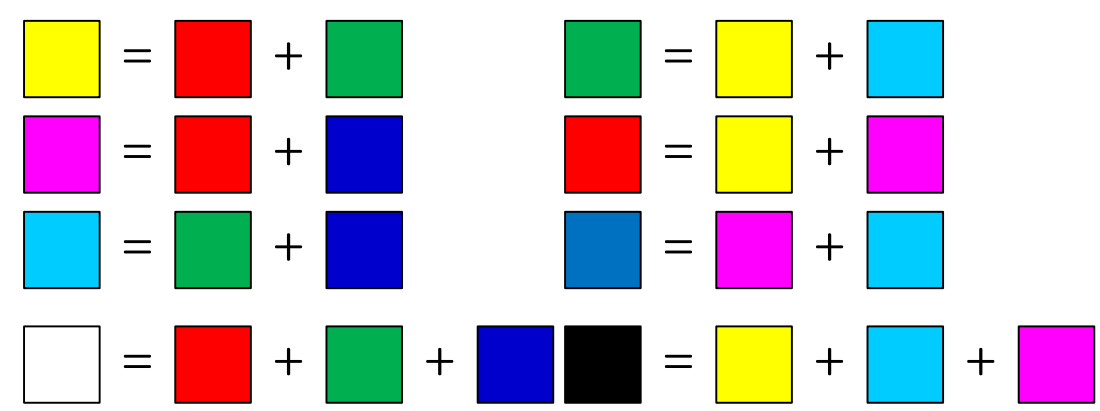

(a)

(b)

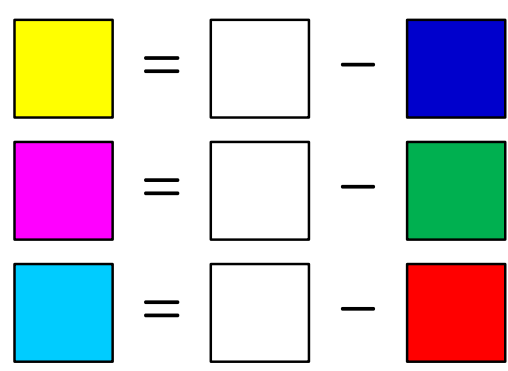

(c)

Figure 1. (a) Additive Color, (b) Subtractive Color, (c) Alternative Method to Generate Yellow, Magenta, and Cyan

\section{Proposed Noise Model}

Generally speaking, image noise can be explained as a random variation of luminosity. The image noise can be generated by three sources: image CCD/CMOS sensor, scanner, and digital camera. In literature, various image noise models have been proposed. Their assumption are shown below:

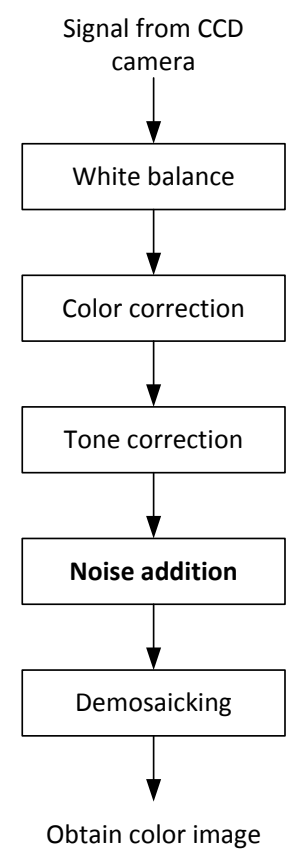

(a)

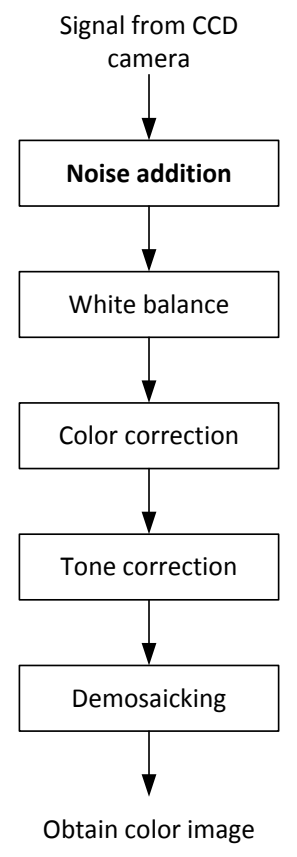

(b)

Figure 2. Comparison of Two Noise Model: (a) Conventional Noise Model, (b) Proposed Noise Model 
Assume 1:

Assume 2:

Sensor noise to be signal-dependent, being proportional to the signal magnitude plus a base level. Give noise arbitrary: $\left(\mathrm{k}_{0}, \mathrm{k}_{1}\right)=$ $(0,0),(25,0),(25,0.1),(25,0.2) .[14,15]$

Assume 3:

An assumption with signal-independent stationary noises of known variances $\sigma_{\mathrm{R}}: \sigma_{\mathrm{G}}: \sigma_{\mathrm{B}}=13: 12: 10$. $[16,17]$

However, each model is based on tentative assumption or observation [18-25]. We assume the most reliable noise model should be the noise which is located before the white-balance, gamma-correction stages, and it should be signal-dependent and signalindependent white Gaussian noise. Moreover, the variance on three primary colors must be different $\left(\sigma_{\mathrm{R}}: \sigma_{\mathrm{G}}: \sigma_{\mathrm{B}}\right)$ in each channels.

Our suggestion is to use other $\left(\sigma_{\mathrm{R}}: \sigma_{\mathrm{G}}: \sigma_{\mathrm{B}}=1.8: 0.7: 1.0\right)$ variance combination. These ratio is obtained empirically. One of important conditions is that $\sigma_{\mathrm{G}}$ should be the smallest, while $\sigma_{\mathrm{B}}$ should be biggest. The reason behind this idea is that the human eye is most sensitive to green, and the least sensitive to blue. Figure 2 shows two noise models. The model in Figure. 2(a) is conventional noise model which is widely used for image denoising applications. In contrast, noise model in Figure. 2(b) is proposed model.

Some results of Figure. 2(a) and 2(b) are shown in Figure. 3. Noisy image shown in Figure. 3(a) is the 1st image of Kodak dataset. Figure 3(b) shows the noisy image of $24^{\text {th }}$ Kodak dataset.

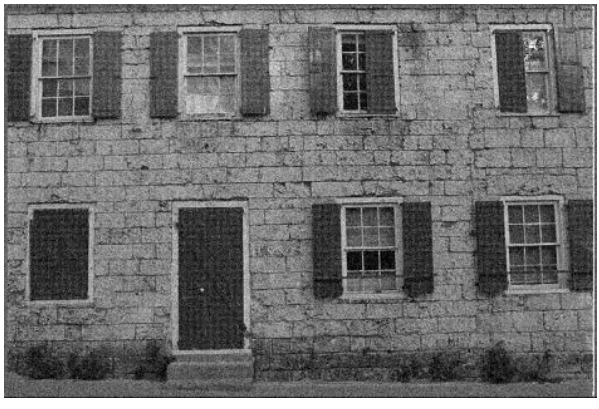

(a)

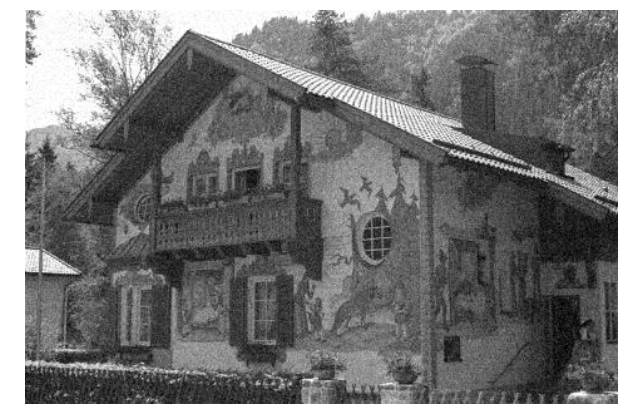

(b)

Figure 3. Generated Noise Image with $\sigma=20$. (a) 1st Kodak Image, (b) 24th Kodak Image

\section{Possible Color Combination of Bayer Pattern}

A Bayer color filter array (CFA) is widely adopted for digital camera. The Bayer filter alignment is a CFA for settling RGB color channels on a square grid of electronic sensors. The Bayer pattern alignment is utilized in most digital image sensors used in digital cameras. A pair of Bayer CFA pattern has four pixels and CFA pattern may be three candidates: RRGB, RGGB, and RGBB. For example, if RGGB is obtained, it has 2 green pixels, 1 red pixel and 1 blue pixel. 


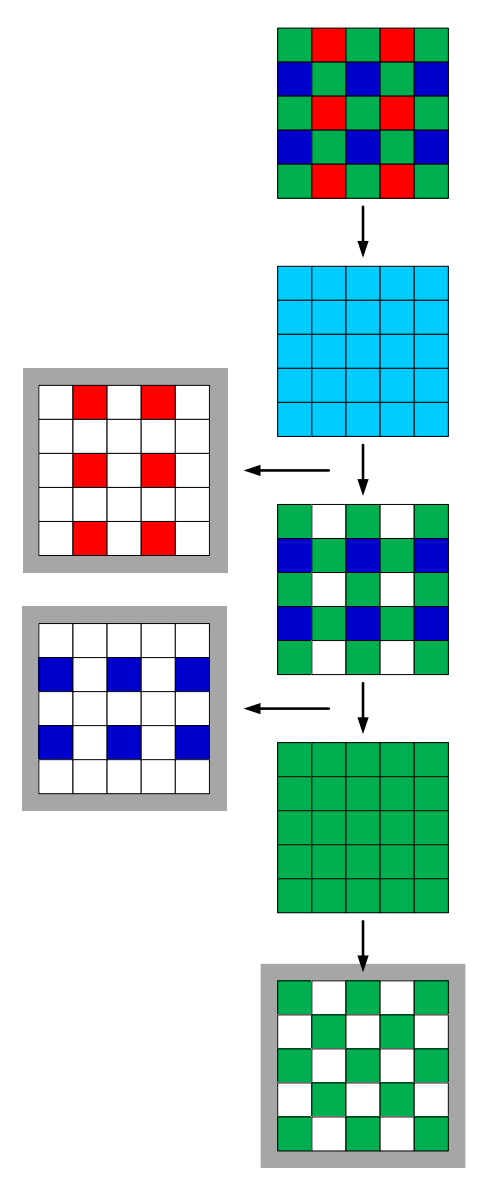

(a)

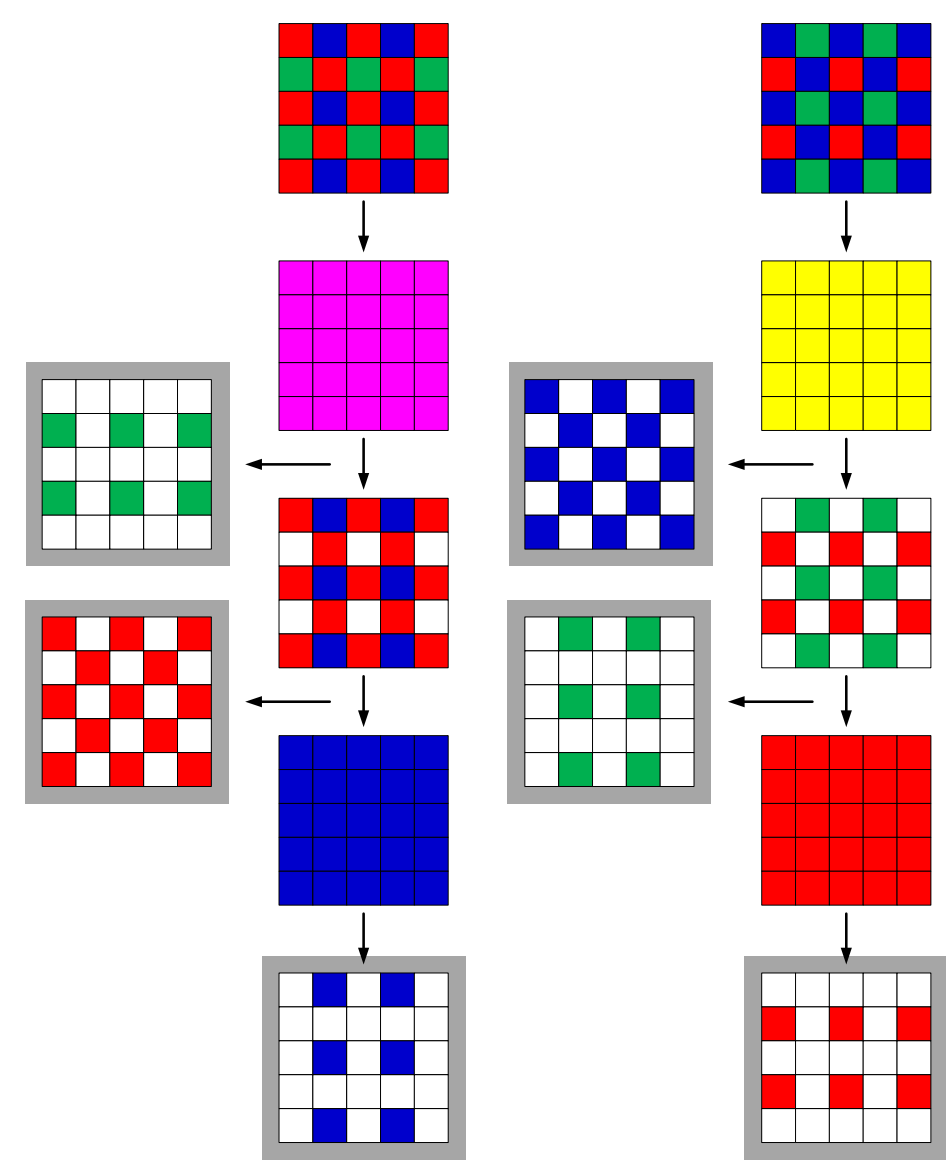

(b) (c)

Figure 4. Process of CFA Pattern Generation: (a) RGGB, (b) RRGB, and (c) RGBB

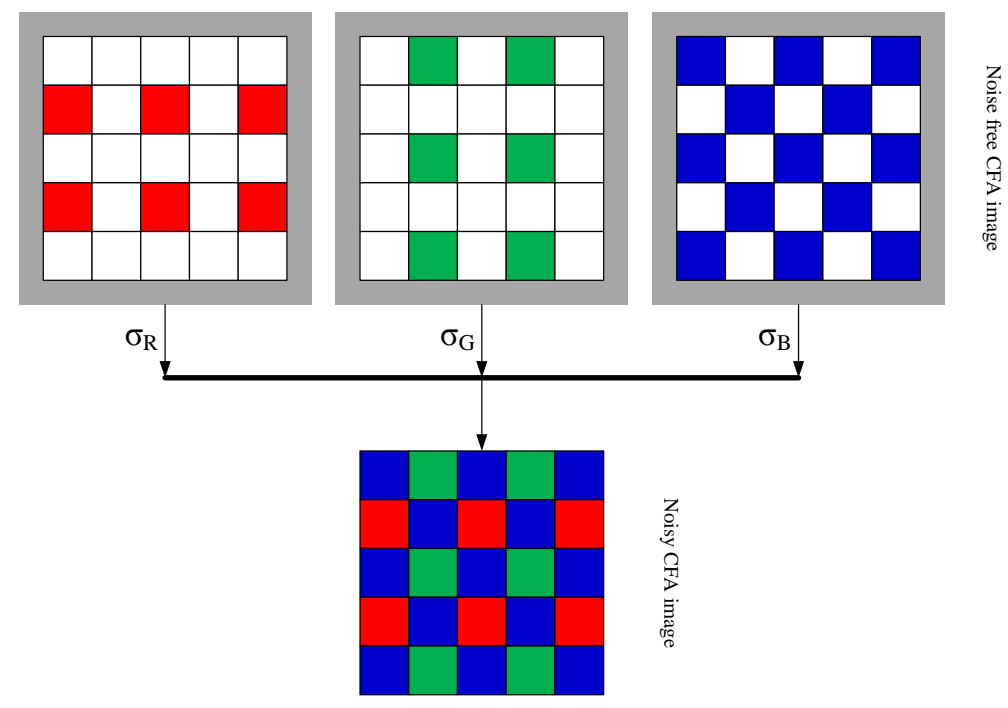

Figure 5. Process of Generating Noisy Image with Variance Level $\sigma_{R}, \sigma_{G}$, and $\sigma_{B}$

Figure 4(a) shows the process of obtaining RGGB patterned CFA. As we can see, by applying cyan filter, red channel is obtained. After that, by using green filter, blue channel 
is obtained. The rest of signals are green channel. In the same manner, RRGB case (Figure. 4b) and RGBB case (Figure. 4c) are explained. Figure 5 shows the process of generating noisy image with variance level $\sigma_{\mathrm{R}}, \sigma_{\mathrm{G}}$, and $\sigma_{\mathrm{B}}$.

\section{Simulation Results}

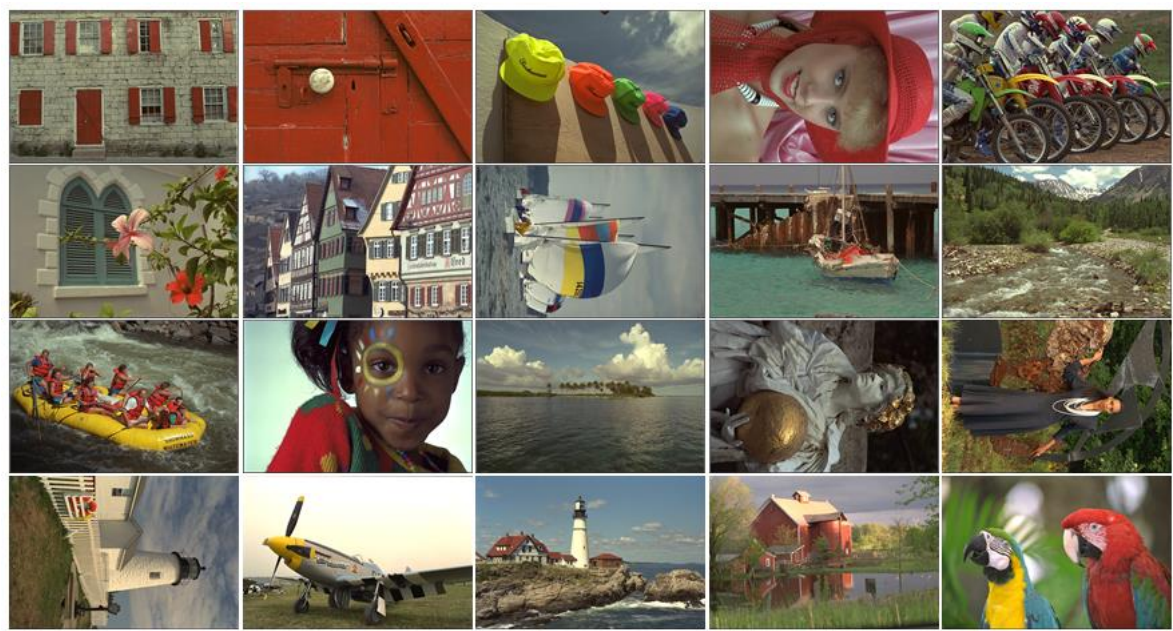

Figure 6. Kodak Dataset

The proposed noise model was tested on 20 Kodak dataset $(1-9,11,13-23)$. Their images are shown in Figure. 6. Table 1 shows PSNR results for two conventional models and the proposed model. Note that assumption 2 is not applicable as $\left\{\left(\mathrm{k}_{0}, \mathrm{k}_{1}\right)=(0,0),(25,0)\right.$, $(25,0.1),(25,0.2)\}$ parameter set is not available for comparison.

Figure 7 and 8 show demosaicked images on generated noisy CFA images by Assumptions 1 and 2, and the proposed model. Applied noise level is $10(\sigma=20)$.

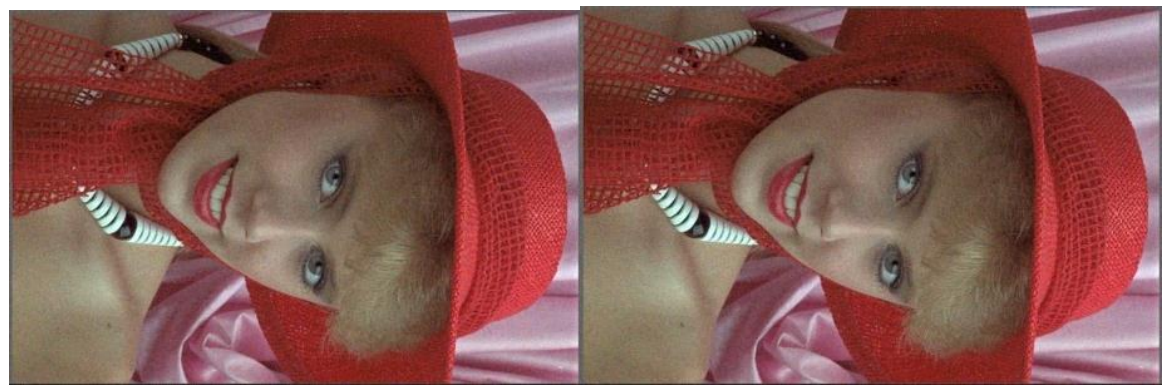

(a)

(b)

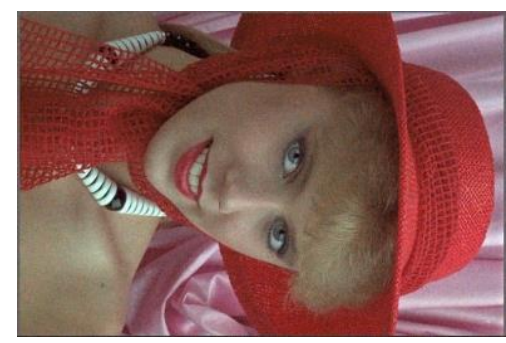

(c)

Figure 7. Demosaicked Images (\#4) on Generated Noisy CFA Images: (a) Assumption 1, (b) Assumption 2, and (c) Proposed Noise Model 


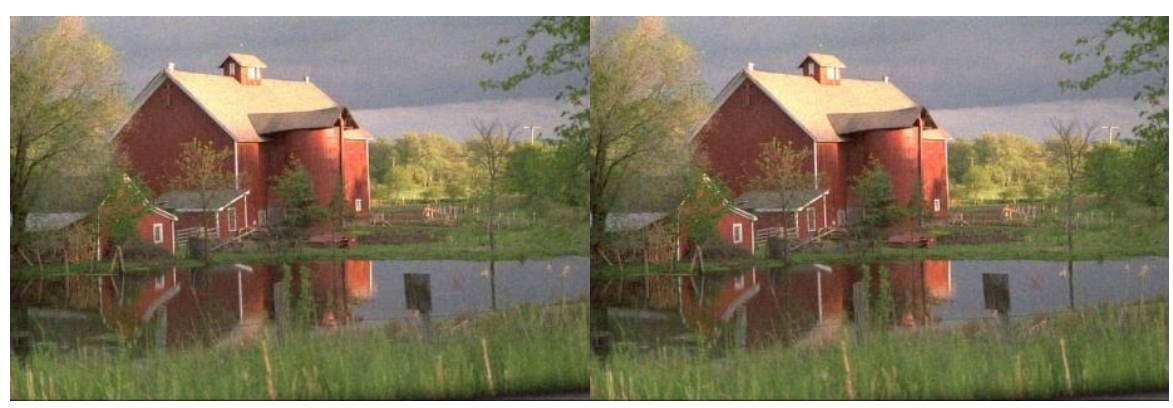

(a)

(b)

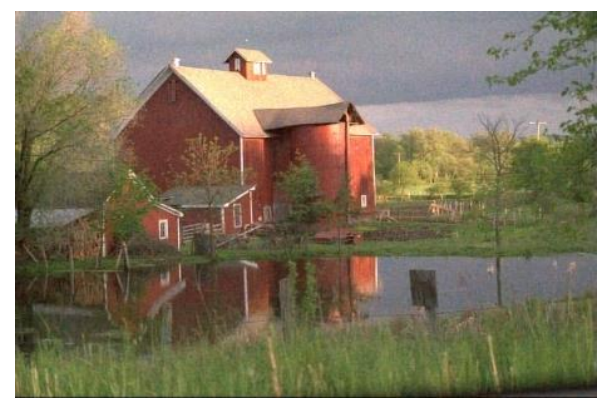

(c)

Figure 8. Demosaicked Images (\#22) on Generated Noisy CFA Images: (a) Assumption 1, (b) Assumption 2, and (c) Proposed Noise Model

Table 1. PSNR Results for Two Conventional Methods and the Proposed Method for 20 Kodak Images

\begin{tabular}{|c|r|r|r|}
\hline Image number & Assumption 1 & Assumption 3 & Proposed model \\
\hline 1 & 36.211 & 36.211 & 36.026 \\
\hline 2 & 23.061 & 23.210 & 22.693 \\
\hline 3 & 14.245 & 14.349 & 13.892 \\
\hline 4 & 30.097 & 30.637 & 29.916 \\
\hline 5 & 19.683 & 19.637 & 19.700 \\
\hline 7 & 28.965 & 29.073 & 29.000 \\
\hline 8 & 43.808 & 43.808 & 43.808 \\
\hline 9 & 34.777 & 34.030 & 34.265 \\
\hline 11 & 19.876 & 20.046 & 19.011 \\
\hline 13 & 28.894 & 28.336 & 29.259 \\
\hline 14 & 21.213 & 21.285 & 21.427 \\
\hline 15 & 20.706 & 20.830 & 20.637 \\
\hline 16 & 43.808 & 43.808 & 43.808 \\
\hline 17 & 34.777 & 34.030 & 34.146 \\
\hline 18 & 29.146 & 29.336 & 28.429 \\
\hline 19 & 27.706 & 27.814 & 27.787 \\
\hline 20 & 23.089 & 23.229 & 22.980 \\
\hline 21 & 36.026 & 36.211 & 36.606 \\
\hline 22 & 49.828 & 49.828 & 49.828 \\
\hline 23 & 27.653 & 27.601 & 27.925 \\
\hline Average & 29.678 & 29.666 & 29.557 \\
\hline
\end{tabular}


Figure 9 shows generated noisy CFA images with various RGB patterns. Figure 9(a) uses RRGB pattern, therefore twice more red information are given for CFA images. In the same matter, Figures 9(b) and (c) uses RGGB and RGBB patterns. Figure 10 shows generated noisy CFA images on RGGB pattern. There scenarios were used: Assumption 1 with $\sigma=10$, Assumption 3 with $\sigma=10$, and the proposed method. As we can see, the proposed noise model reflected the most natural images when it compared with other images.

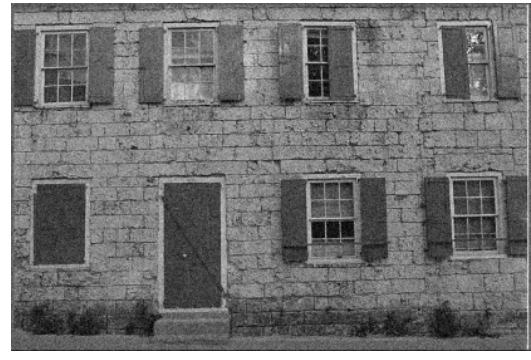

(a)

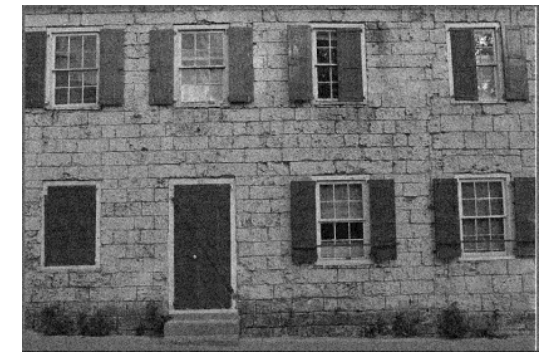

(b)

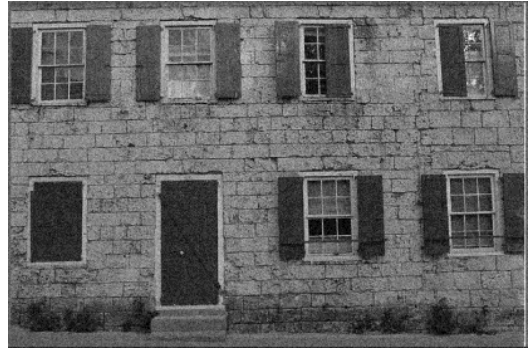

(c)

Figure 9. Generated Noisy CFA Images: (a) RRGB, (b) RGGB, and (c) RGBB

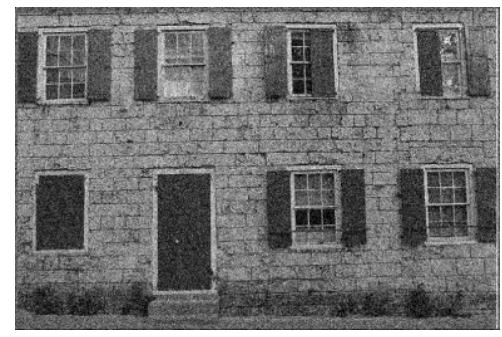

(a)

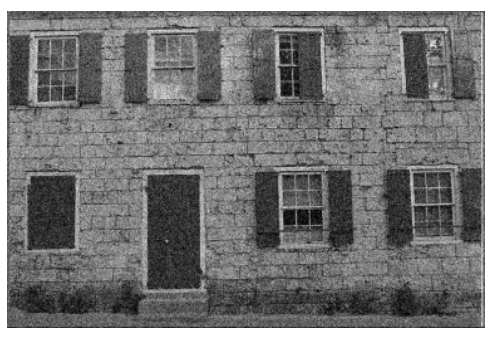

(b)

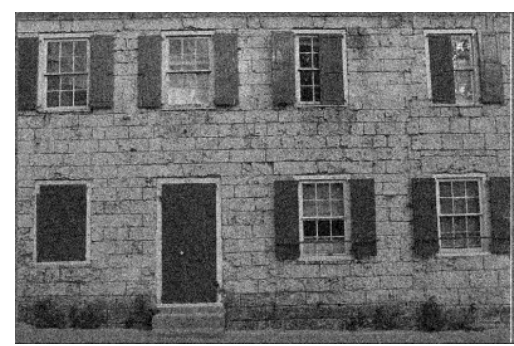

(c)

Figure 10. Generated Noisy CFA Images Using RGGB Pattern: (a) Assumption $1(\sigma=10)$, (b) Assumption $3(\sigma=10)$, and (c) Proposed Noise Model 


\section{Conclusions}

In this paper, we studied a new noise model for conventional Bayer color filter array. Performance of three color patterns were evaluated and compared. Conventional noise models do not consider some important properties such as white balance, gamma and tone correction. The proposed noise model suggested using above issues. Experimental results revealed that the proposed noise model is more acceptable to noisy demosaicking.

\section{Acknowledgements}

This work was supported by the National Research Foundation of Korea (NRF) Grant funded by the Korean Government (MSIP) (2014025627).

\section{Footnote}

This paper is a revised and expanded version of a paper entitled "Noise Model Analysis for Sensor Arrays" presented at "The International Conference on Computer and Computing Science 2014".

\section{References}

[1] Leon G., "Probability and Random Processes for Electrical Engineering," vol. 2.

[2] Lee J. S., Kuo Y. M., Chung P. C. and Chen E. L, "Naked image detection based on adaptive and extensible skin color model," Pattern Recognition, vol. 40 no. 8, (2007), pp. 2261-2270.

[3] T. T. Ng, S. F. Chang, J. Hsu, L. Xie and M. P. Tsui, "Physics-motivated features for distinguishing photographic images and computer graphics, in ACM Multimedia," Singapore, November, (2005).

[4] S. Lyu and H. Farid, "How realistic is photorealistic," IEEE Transactions on Signal Processing, vol. 53, February (2005), pp. 845-850.

[5] Yin X. and Xie M., "Estimation of the fundamental matrix from uncalibrated stereo hand images for 3D hand gesture recognition," Pattern Recognition, vol. 36 no. 3, (2002), pp. 567-584.

[6] A. Vailaya, A. K. Jain and H. J Zhang, "On image classification: city vs. landscapes," Intl. J. Pattern Recognition, vol. 31, (1998), 1921-1936.

[7] T. Ianeva, A. D. Vries and H. Rohrig, "Detecting cartoons: a case study in automatic video-genre classification," in IEEE International Conference on Multimedia and Expro, vol. 1, (2003), pp. 449-452.

[8] Wu Y., He H.; Ru T. and Zheng D., "Hand Segmentation for Augmented Reality System," Digital Media and its Application in Museum \& Heritages, Second Workshop, vol., no., 10-12 December. (2007), pp. 395-401.

[9] Florin C., Riad H. and Alex L., "Distinguishing paintings from photographs," Computer Vision and Image Understanding, vol. 100, (2005), pp. 249-273.

[10] A. R. Smith, "Color gamut transform pairs," Computer. Graph, vol. 12 no. 3, (1978), pp. 12-19.

[11] M. Weinberger, G. Seroussi and G. Sapiro, "LOCO-I: A low complexity content-based lossless image compression algorithm," Proc. of IEEE Data Compression Conf., (1996), pp. 140-149.

[12] B. Bayer, “Color imaging array,” U.S. Patent 3971065, July (1976).

[13] D. Paliy, V. Katkovnik, R. Bilcu, S. Alenius and K. Egiazarian, "Spatially adaptive color filter array interpolation for noiseless and noisy data," International Journal of Imaging Systems and Technology, vol. 17 no. 3, October (2007), pp. 105-122.

[14] K. Hirakawa and T. W. Parks, "Adaptive homogeneity-directed demosaicing algorithm," IEEE Trans. Image processing, vol. 14 no. 3, (1997), pp. 360-369.

[15] K. Hirakawa and T. W. Parks, "Adaptive homogeneity-directed demosaicing algorithm," IEEE Transactions on Image Processing, vol. 14, no. 3, (2005), pp. 360-369.

[16] L. Zhang, X. Wu and D. Zhang, "Color reproduction from noisy CFA data of single sensor digital cameras,” IEEE Trans. Image Process., vol. 16 no. 9, September (2007), pp. 2184-2197.

[17] L. Zhang, R. Lukac, X. Wu and D. Zhang, "PCA-based spatially adaptive denoising of CFA images for single-sensor digital cameras," IEEE Trans. Image Process., vol. 18 no. 4, April (2009), pp. 797-812.

[18] Cong J., Qing H. D. and Jun L., "Computer Virus Propagation Model Based on Variable Propagation Rate," IJAST, vol. 1, December (2008), pp. 29-34.

[19] Ching T. H., Yeh K. W. and Kuo M. H., "Hybrid Watermarking Scheme for Halftone Images," IJAST, vol. 1, December (2008), pp. 9-20.

[20] W. Yun, S. Lee and G. Jeon., "Noise Model Analysis for Sensor Arrays," in Proc. COMCOMS 2014, December (2014).

[21] G. Jeon, M. Anisetti, V. Bellandi and J. Jeong, "Fuzzy rule-based edge-restoration algorithm in HDTV interlaced sequences,” IEEE Trans. Consumer Electronics, vol. 53 no. 2, May (2007), pp. 725-731. 
[22] G. Jeon, M. Anisetti, V. Bellandi, E. Damiani and J. Jeong, "Rough sets-assisted subfield optimization for alternating current plasma display panel," IEEE Trans. Consumer Electronics, vol. 53 no. 3, August (2007), pp. 825-832.

[23] G. Jeon, M. Anisetti, V. Bellandi, E. Damiani and J. Jeong, "Fuzzy weighted approach to improve visual quality of edge-based filtering," IEEE Trans. Consumer Electronics, vol. 53 no. 4, November (2007), pp. 1661-1667.

[24] G. Jeon, M. Anisetti, J. Lee, V. Bellandi, E. Damiani and J. Jeong, "Concept of linguistic variable-based fuzzy ensemble approach: application to interlaced HDTV sequences," IEEE Trans. Fuzzy Systems, vol. 17 no. 6, December (2009), pp. 1245-1258.

[25] G. Jeon, M. Y. Jung, M. Anisetti, V. Bellandi, E. Damiani and J. Jeong, "Specification of the geometric regularity model for fuzzy if-then rule based deinterlacing," IEEE/OSA Journal of Display Technology, vol. 6 no. 6, June (2010), pp. 235-243.

\section{Author}

Gwanggil Jeon, received the BS, MS, and $\mathrm{PhD}$ (summa cum laude) degrees in Department of Electronics and Computer Engineering from Hanyang University, Seoul, Korea, in 2003, 2005, and 2008, respectively.

From 2008 to 2009, he was with the Department of Electronics and Computer Engineering, Hanyang University, from 2009 to 2011, he was with the School of Information Technology and Engineering (SITE), University of Ottawa, as a postdoctoral fellow, and from 2011 to 2012, he was with the Graduate School of Science \& Technology, Niigata University, as an assistant professor. He is currently an assistant professor with the Department of Embedded Systems Engineering, Incheon National University, Incheon, Korea. His research interests fall under the umbrella of image processing, particularly image compression, motion estimation, demosaicking, and image enhancement as well as computational intelligence such as fuzzy and rough sets theories.

He was the recipient of the IEEE Chester Sall Award in 2007 and the 2008 ETRI Journal Paper Award. 
International Journal of Multimedia and Ubiquitous Engineering

Vol.10, No.7 (2015) 